\title{
Identification of two mutations increasing the methanol tolerance of Corynebacterium glutamicum
}

\author{
Lennart Leßmeier and Volker F. Wendisch ${ }^{*}$ (D)
}

\begin{abstract}
Background: Methanol is present in most ecosystems and may also occur in industrial applications, e.g. as an impurity of carbon sources such as technical glycerol. Methanol often inhibits growth of bacteria, thus, methanol tolerance may limit fermentative production processes.

Results: The methanol tolerance of the amino acid producing soil bacterium Corynebacterium glutamicum was improved by experimental evolution in the presence of methanol. The resulting strain Tol1 exhibited significantly increased growth rates in the presence of up to $1 \mathrm{M}$ methanol. However, neither transcriptional changes nor increased enzyme activities of the linear methanol oxidation pathway were observed, which was in accordance with the finding that tolerance to the downstream metabolites formaldehyde and formate was not improved. Genome sequence analysis of strain Tol1 revealed two point mutations potentially relevant to enhanced methanol tolerance: one leading to the amino acid exchange A165T of O-acetylhomoserine sulfhydrolase MetY and the other leading to shortened CoA transferase Cat (Q342*). Introduction of either mutation into the genome of C. glutamicum wild type increased methanol tolerance and introduction of both mutations into C. glutamicum was sufficient to achieve methanol tolerance almost indistinguishable from that of strain Tol1.
\end{abstract}

Conclusion: The methanol tolerance of C. glutamicum can be increased by two point mutations leading to amino acid exchange of O-acetylhomoserine sulfhydrolase MetY and shortened CoA transferase Cat. Introduction of these mutations into producer strains may be helpful when using carbon sources containing methanol as component or impurity.

Keywords: Corynebacterium, Methanol, Tolerance, O-acetylhomoserine sulfhydrolase, Coenzyme A hydrolase/transferase, Methionine, Ethanol, Acetyl-CoA, MetY, Cat

\section{Background}

Methanol naturally occurs in most ecosystems and is the second most abundant organic gas in the atmosphere besides methane [1]. The major source for methanol is the emission by plants [2]. Also the decay of plants, biomass burning or atmospheric oxidation of methane give rise to methanol [3]. One example of microbial production of methanol in nature is the pectin degradation by Clostridium butyricum [4].

Methanol itself is cytotoxic since it affects the fluidity of cellular membranes and alters their mechanical

\footnotetext{
* Correspondence: volker.wendisch@uni-bielefeld.de Genetics of Prokaryotes, Faculty of Biology \& Center for Biotechnology, Bielefeld University, Bielefeld 33615, Germany
}

stability [5]. Membrane disruption has been reported for incubation with more than $44 \%$ methanol [6]. Also indirect toxic effects related to methanol have been reported, mostly due to accumulation of the methanol degradation products formaldehyde and formate. Formaldehyde is a potent cytotoxin due to its high reactivity with proteins and DNA [7, 8]. In mammalian species, toxicity of methanol is mainly attributed to an accumulation of formate, causing metabolic acidosis [9]. Formate has also been demonstrated to inhibit mitochondrial cytochrome oxidase of mammals [10, 11]. Additionally, the oxidation of methanol and its metabolites is often accompanied by the generation of superoxide anions, which give rise to oxidative stress and may be involved in lipid peroxidation $[12,13]$. 
According to its high abundance, degradation of methanol is a common feature in nature. Besides detoxification, methanol can also be utilized as a carbon and energy source by a wide variety of eukaryotic and prokaryotic methylotrophs [14]. Metabolism of methanol is typically initiated by its oxidation, which can be catalyzed by many different enzymes such as pyrroloquinoline quinone (PQQ)-dependent methanol dehydrogenase e.g. in Methylobacterium extorquens [15], class I alcohol dehydrogenase e.g. in humans [16] or alcohol oxidase e.g. in Candida boidinii [17].

The resulting formaldehyde is a branching point between detoxification pathways and the assimilation pathways in methylotrophs. In non-methylotroph organisms, the detoxification of formaldehyde typically occurs in linear pathways, in which formaldehyde is oxidized to formate by formaldehyde dehydrogenase and further to carbon dioxide catalyzed by formate dehydrogenase [14].

The Gram-positive bacterium Corynebacterium glutamicum belongs to the mycolic acid-containing actinomycetes and is particularly known for its use in the millionton-scale production of amino acids [18, 19]. Recent studies on this organism revealed that C. glutamicum possesses an endogenous pathway for the oxidation of methanol to carbon dioxide. In this pathway, the oxidation of methanol to formaldehyde is mainly performed by the alcohol dehydrogenase AdhA (cg3107), but at least one additional enzyme of hitherto unknown identity is also involved [20]. Formaldehyde is oxidized by two distinct enzymes, the acetaldehyde dehydrogenase Ald (cg3096) and the mycothiol-dependent formaldehyde dehydrogenase FadH (cg0387) [21]. The resulting formate is subsequently converted to carbon dioxide by formate dehydrogenase FdhF (cg0618) also involving the gene products encoded by cg0616 and cg0617 [22]. The electron acceptor of Fdh is currently unknown.

Methanol tolerance can be a bottleneck in industrial biotechnology if the culture broth contains methanol, either as a part of the process or an impurity e.g. of the carbon source. For example, Gluconobacter frateurii needed to be adapted to high methanol concentrations before it could be cultivated using methanol-containing raw glycerol as a substrate, which occurs as a byproduct during biodiesel production [23]. Additionally, methanol represents an interesting upcoming carbon source for microbial production of chemicals [24, 25] e.g. cadaverine [26]. C. glutamicum cannot use methanol as sole carbon source [20,21], although engineered strains do convert methanol to a certain degree to intracellular metabolites [27] and to products such as cadaverine [28].

However, C. glutamicum has been engineered to use pure glycerol for growth and amino acid production [29], while certain technical qualities of glycerol obtained from bio-diesel factories, which contain methanol as impurity, were inhibitory [30]. The aim of this study was to improve the methanol tolerance of C. glutamicum. Genome sequence analysis of an evolved strain and subsequent genetic and physiological experiments revealed that two single nucleotide polymorphisms (SNPs) significantly increased the tolerance to methanol of $C$. glutamicum without directly affecting the methanol detoxification pathway.

\section{Results}

Biphasic, non-linear growth response of C. glutamicum wild type to methanol

In accordance with operation of linear methanol detoxification in C. glutamicum [21, 22], this bacterium is able to grow in the presence of up to $1.3 \mathrm{M}$ methanol reaching high biomass concentrations [20]. When C. glutamicum wild-type strain ATCC 13032 carrying the vector pVWEx1 was grown in the presence of a wide range of methanol concentrations, however, the growth rate showed a non-linear dependency on methanol (Fig. 1). In a first concentration range up to $120 \mathrm{mM}$ methanol, a sharp decrease of the growth rate was observed with a growth rate in the presence of $120 \mathrm{mM}$ methanol decreased by $30 \%\left(0.30 \pm 0.01 \mathrm{~h}^{-1}\right)$ as compared to growth without methanol $\left(0.43 \pm 0.00 \mathrm{~h}^{-1}\right)$. In the second concentration range from $480 \mathrm{mM}$ to $3 \mathrm{M}$ methanol, the growth rate gradually decreased but with a much smaller slope as e.g. increasing the methanol concentration eight fold from $120 \mathrm{mM}$ to $960 \mathrm{mM}$ only reduced the growth rate from $0.30 \pm 0.01 \mathrm{~h}^{-1}$ to $0.25 \pm 0.03 \mathrm{~h}^{-1}$ (Fig. 1). The bi-phasic, non-linear dependence of the growth rate on the presence of methanol may indicate that the effect of methanol on growth of C. glutamicum may be more complex than anticipated.

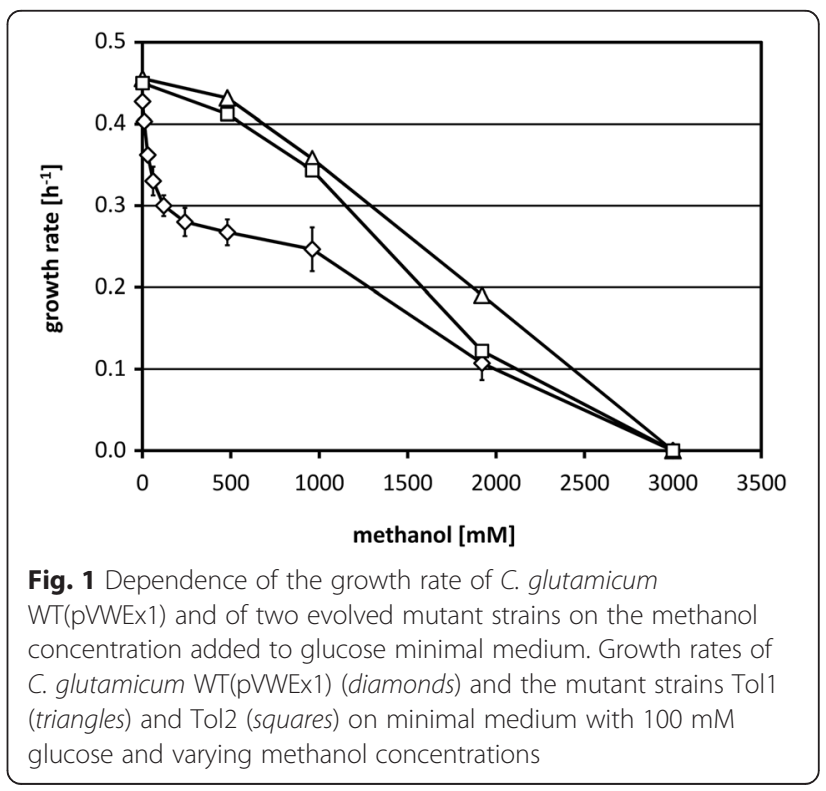




\section{Adaptive laboratory evolution of C. glutamicum in the presence of methanol}

Experimental evolution in selective medium was used to achieve a genetic adaption to the presence of methanol and thereby increasing the methanol tolerance of C. glutamicum. For this purpose, repeated cultivations with the wild-type strain ATCC 13032 carrying the vector pVWEx1 were performed in minimal medium containing $120 \mathrm{mM}$ methanol. After this selection process, growth of two independently isolated strains, named Tol1 and Tol2, was analyzed in the presence and absence of methanol. In the absence of methanol, strains Tol1 and Tol2 and the parental strain C. glutamicum (pVWEx1) showed similar growth behavior with a growth rate of $0.44 \pm 0.01 \mathrm{~h}^{-1}$ in glucose minimal medium. A comparison of the growth behavior at diverse methanol concentrations revealed that the methanol tolerant strains showed significantly increased growth rates at concentrations up to $1 \mathrm{M}$, while growth in the presence of $3 \mathrm{M}$ methanol was neither observed for the evolved strains nor for the parental strain (Fig. 1). Interestingly, unlike the wild type, the dependence of the growth rate on methanol was almost linear for the tolerant strains (Fig. 1), and lower methanol concentrations did not cause severe growth retardation of the tolerant strains. Because both mutant strains showed a similar phenotype, only Tol1 was used in further analyses to identify the mutation(s) overcoming the strong growth impairment by low methanol concentrations.

\section{Role of the linear methanol detoxification pathway}

C. glutamicum possesses the linear methanol detoxification pathway involving oxidation of methanol via formaldehyde and formate to carbon dioxide. In order to test whether methanol oxidation by Tol1 differs from $C$. glutamicum wild type, enzyme activity of alcohol dehydrogenase AdhA was determined. The specific alcohol dehydrogenase activities with ethanol as substrate were comparable for C. glutamicum wild type (79 \pm $2 \mathrm{mU} / \mathrm{mg}$ ) and Tol1 (78 $\pm 1 \mathrm{mU} / \mathrm{mg})$ grown in LB. Under inducing conditions [20, 31, 32], i.e. after growth in LB with $325 \mathrm{mM}$ ethanol, alcohol dehydrogenase activities increased in C. glutamicum wild type (183 \pm $18 \mathrm{mU} / \mathrm{mg}$ ) and Tol1 (276 $\pm 27 \mathrm{mU} / \mathrm{mg})$. Moreover, as growth of an $a d h A$ deletion mutant in glucose minimal medium with up to $960 \mathrm{mM}$ methanol was comparable to that of C. glutamicum wild type (data not shown), AdhA did not contribute notably to the response of $C$. glutamicum to methanol.

Methanol oxidation gives rise to the highly toxic metabolite formaldehyde. In glucose minimal medium without formaldehyde, the growth rates were comparable for C. glutamicum WT(pVWEx1) $\left(0.41 \pm 0.01 \mathrm{~h}^{-1}\right)$ and Tol1 $\left(0.42 \pm 0.01 \mathrm{~h}^{-1}\right)$ and they were decreased similarly to $0.32 \pm 0.01 \mathrm{~h}^{-1}$, when formaldehyde was present (Additional file 1: Fig. S1a). In addition, the specific activities of formaldehyde oxidizing enzymes Ald and $\mathrm{FadH}$ determined by an in vivo assay (Additional file 1: Figure S1b) were comparable for both strains

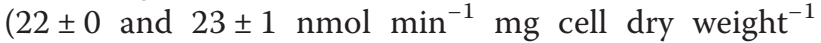
for Tol1 and WT(pVWEx1), respectively). In addition, growth of C. glutamicum Tol1 and WT(pVWEx1) in the presence of $200 \mathrm{mM}$ potassium formate, the second potentially toxic intermediate of the linear methanol oxidation pathway, was comparable (each $0.24 \pm 0.01 \mathrm{~h}^{-1}$, data not shown). Thus, C. glutamicum Tol1 showed improved tolerance to methanol, but neither to formaldehyde nor to formate.

\section{DNA microarray analysis of global gene expression of $C$. glutamicum Tol1}

Genome-wide gene expression analyses using microarrays were performed in order to identify differentially expressed genes in strain Tol1, which might contribute to methanol tolerance. In a first experiment, mRNA levels of Tol1 and C. glutamicum WT(pVWEx1) were compared during exponential growth in complex medium. The genes cysK and met $Y$ which are involved in amino acid metabolism and the prpD2B2C2 operon showed higher mRNA levels in Tol1 than in the parental strain (Additional file 1: Table S1). The prpD2B2C2 operon which codes for enzymes required for propionate metabolism shows a strong induction in presence of propionate [33]. To analyze if the increased expression of this operon positively affects growth with methanol, C. glutamicum wild type was grown in glucose minimal medium with $0 \mathrm{mM}$ or $7 \mathrm{mM}$ propionate and $120 \mathrm{mM}$ methanol was added after two hours. Methanol decreased the growth rate by about $45 \%$ in the presence or absence of propionate (Additional file 1: Fig. S2), demonstrating that the induction of the prpD2B2C2 operon did not increase the tolerance to methanol.

In a second experiment, global gene expression changes of C. glutamicum Tol1 and WT due to addition of methanol were compared. C. glutamicum WT was cultivated in glucose minimal medium with/without addition of $30 \mathrm{mM}$ methanol. To avoid growth ratedependent differences, the methanol tolerant strain Tol1 was cultivated in the presence of $120 \mathrm{mM}$ methanol, a methanol concentration leading to a comparable growth rate reduction. As consequence of methanol addition, expression of 35 and 31 genes, respectively, changed in C. glutamicum Tol1 and WT, respectively (Additional file 1: Table S2). Expression of only three genes, namely $a d h A$ and two genes for hypothetical proteins (cg1625 and cg1291; Fig. 2), changed in both strains. It was observed that expression of genes responsible for ethanol utilization via acetate in the glyoxylate cycle ( $a d h A$, ace $A$ 


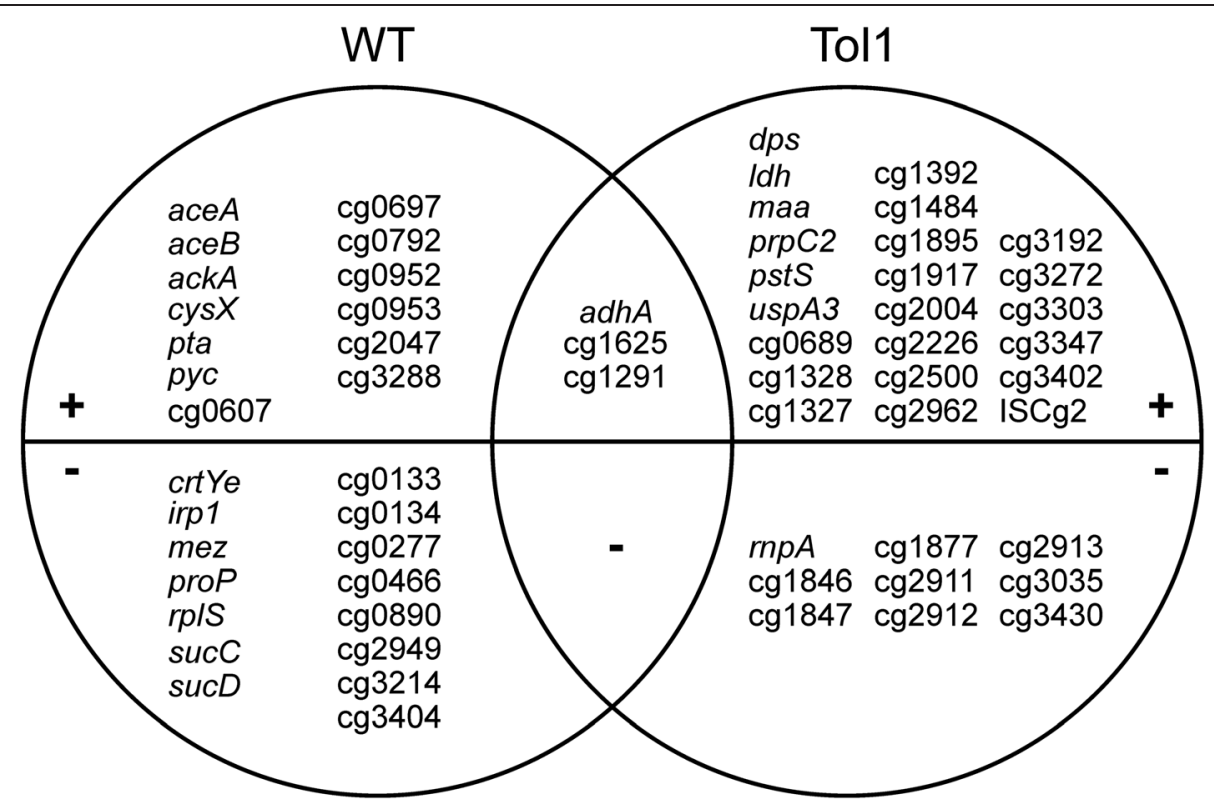

Fig. 2 Venn diagram showing gene expression changes in a comparison of C. glutamicum Tol1 with wild type. The strains were cultivated in minimal medium with $100 \mathrm{mM}$ glucose in the presence or absence of methanol. Genes with increased mRNA levels in the presence of methanol are shown in the $(+)$ section and genes with reduced mRNA levels are shown in the $(-)$ section of the graph

for isocitrate lyase, $a c e B$ for malate synthase, $a c k A$ for acetate kinase, $p t a$ for phosphate acetyltransferase and sucCD for succinyl-CoA synthetase) were induced in the wild type, but not in Tol1. Since expression of these genes is activated in acetate minimal medium by transcriptional activator RamA [34-36], growth of C. glutamicum $\triangle \mathrm{ramA}$ in the presence of methanol was analyzed (Additional file 1: Fig. S3). However, growth of C. glutamicum $\triangle \operatorname{ramA}$ and of the wild type in glucose minimal medium was similar without methanol $(0.36 \pm$ $0.00 \mathrm{~h}^{-1}$ vs. $\left.0.39 \pm 0.00 \mathrm{~h}^{-1}\right)$ and with $480 \mathrm{mM}$ methanol $\left(0.23 \pm 0.00 \mathrm{~h}^{-1}\right.$ vs. $\left.0.25 \pm 0.00 \mathrm{~h}^{-1}\right)$.

\section{Genome sequencing of Tol1 and introduction of mutations into the wild-type genome}

Since neither physiological experiments and enzyme activity measurements nor DNA microarray analysis revealed the mutation(s) responsible for enhanced methanol tolerance of C. glutamicum Tol1, its genome was sequenced. Compared to the published genome sequence of C. glutamicum ATCC 13032 [37], 101 insertions, deletions or SNPs were found (Additional file 1: Table S3). Previously, a similar number of changes has been observed when genomes of C. glutamicum mutants were sequenced $[38,39]$. Therefore, all sequence changes found in the genome of Tol1 were compared to the genome sequence of a control strain derived from the same wild type during the same period of time. Only 29 mutations were unique for Tol1. Of these, one SNP representing a synonymous substitution, four SNPs located in intergenic regions and 19 SNPs in a gene (cg2069) a putative secreted protein of the prophage CGP3 [37] were not considered further.

The remaining five SNPs led to amino acid substitutions: a change of alanine to valine at position 19 (A19V) of conserved hypothetical protein encoded by cg0198, change L328S in the putative membrane protein encoded by cg1245, change $\mathrm{D} 67 \mathrm{H}$ in the ABC-type transporter subunit encoded by cg2204 and change A165T in the O-acetylhomoserine sulfhydrolase MetY. One SNP caused a nonsense mutation (Q342*) and resulted in a truncated version of CoA transferase Cat lacking the $161 \mathrm{C}$-terminal amino acids. These five mutations were introduced individually into the genome of C. glutamicum WT resulting in the strains T0198, T0755, T1245, T2204 and T2840. None of these mutations affected growth in glucose minimal medium or in complex medium (data not shown). Growth of strains T0198, T1245 and T2204 in glucose minimal medium supplemented with $240 \mathrm{mM}$ methanol was comparable to that of the wild type (data not shown). However, strain T0755 showed a slightly increased growth rate $\left(0.32 \pm 0.00 \mathrm{~h}^{-1}\right)$ in glucose minimal medium supplemented with $240 \mathrm{mM}$ methanol as compared to WT $\left(0.31 \pm 0.00 \mathrm{~h}^{-1}\right)$, while strain T2840 grew significantly faster $\left(0.35 \pm 0.00 \mathrm{~h}^{-1}\right)$ (Fig. 3). The mutation of cat observed in Tol1 caused a truncation of the enzyme at position 342, thus, while the N-terminal acetyl-CoA hydrolase/transferase domain (pfam02550) predicted by alignment to the conserved domain database (CDD) [40] 


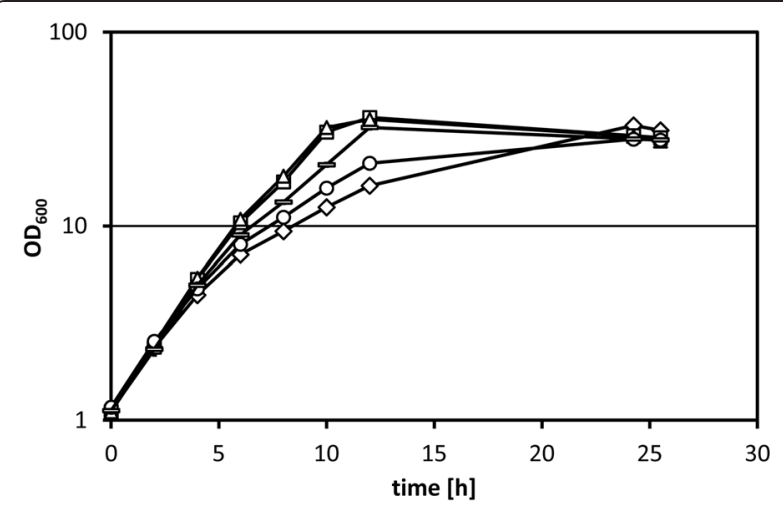

Fig. 3 Growth of various C. glutamicum strains in glucose minimal medium supplemented with $240 \mathrm{mM}$ methanol. Growth of C. glutamicum WT (diamonds), Tol1 (triangles), T0755 (circles), T2840 (bars) and T0755 +2840 (squares) in minimal medium supplemented with $100 \mathrm{mM}$ glucose and $240 \mathrm{mM}$ methanol. Means and standard deviations of two independent cultures are shown

is present, the respective C-terminal domain (pfam13336) is not. This severe modification may have changed its enzymatic (side) activity or caused a loss of enzyme function. To analyze if the latter causes the increased methanol tolerance, a cat deletion strain lacking both the $\mathrm{N}$ - and $\mathrm{C}$ terminal regions of Cat [41] was analyzed. In the absence of methanol, this strain showed a slightly lower growth rate $\left(0.38 \pm 0.00 \mathrm{~h}^{-1}\right)$ than WT $\left(0.39 \pm 0.00 \mathrm{~h}^{-1}\right)$, while the growth rate of $\Delta$ cat $\left(0.29 \pm 0.00 \mathrm{~h}^{-1}\right)$ was significantly higher than that of the WT $\left(0.20 \pm 0.00 \mathrm{~h}^{-1}\right)$ in minimal medium with $100 \mathrm{mM}$ glucose and $240 \mathrm{mM}$ methanol (Additional file 1: Fig. S4). These results indicated that the lack of Cat activity improved methanol tolerance to a comparable extent as observed when Cat was truncated due to the nonsense mutation (Q342*).

When both mutations were combined, the resulting strain T0755 +2840 grew as fast $\left(0.38 \pm 0.00 \mathrm{~h}^{-1}\right)$ as Tol1 in glucose minimal medium supplemented with $240 \mathrm{mM}$ methanol (Fig. 3). Thus, the identified two mutations resulting in amino acid exchange A165T in the $\mathrm{O}$-acetylhomoserine sulfhydrolase MetY and in truncation $\left(\mathrm{Q} 342^{*}\right)$ of CoA transferase were sufficient to explain the improved methanol tolerance of Toll.

\section{Influence of mutations increasing methanol tolerance on growth with ethanol}

C. glutamicum wild type is able to utilize ethanol, but not methanol, as sole carbon source [31]. To determine if one or both of the mutations increasing tolerance to methanol affects growth with ethanol as sole carbon source, growth experiments with C. glutamicum WT, Tol1, T0755, T2840 and T0755 +2840 in minimal medium containing $325 \mathrm{mM}$ ethanol as sole carbon source were performed. Surprisingly, strain Tol1 showed no growth on ethanol, while C. glutamicum WT grew with a growth rate of $0.14 \pm 0.00 \mathrm{~h}^{-1}$ (Fig. 4). Strain T0755 grew with ethanol at a growth rate of $0.16 \pm$ $0.00 \mathrm{~h}^{-1}$, but neither strain T2840 nor strain T0755+ 2840 were able to grow in ethanol minimal medium (Fig. 4). Thus, truncation of CoA transferase Cat due to missense mutation Q342* as present in strains Tol1, T2840 and T0755 + 2840 resulted in the inability to utilize ethanol as sole source of carbon and energy. To test if a cat deletion mutant lacking both the $\mathrm{N}$ - and $\mathrm{C}$ terminal parts of Cat is able to grow, C. glutamicum WT, Tol1, T2840 and $\Delta c a t$ was grown in minimal medium with $1 \%$ ethanol as sole carbon source. While C. glutamicum WT could grow, strains Tol1, T2840 and $\Delta$ cat did not (Additional file 1: Fig. S5).

\section{Discussion}

In this study, the response of C. glutamicum to methanol was characterized to be non-linear involving a sharp decrease of the growth rate in the presence of lower methanol concentrations and a less pronounced decrease evident at methanol concentrations in excess of about $250 \mathrm{mM}$. Genome sequencing of a strain selected by adaptive laboratory evolution identified two SNPs that subsequently were shown to be sufficient to explain increased methanol tolerance of the selected strain. The relevant SNPs led to amino acid substitution A165T in the O-acetylhomoserine sulfhydrolase MetY and in truncation (Q342*) of CoA transferase by 161 amino acids.

Mutations which increase the tolerance to methanol in other organisms are so far reported to be mostly related to enzymes of methanol pathways and thereby reducing the accumulation of toxic downstream metabolites. For example the methanol tolerance of Dictyostelium increased significantly by loss of a catalase, which is supposed to be the main enzyme in this organism oxidizing methanol to formaldehyde [42]. Also methanol tolerance

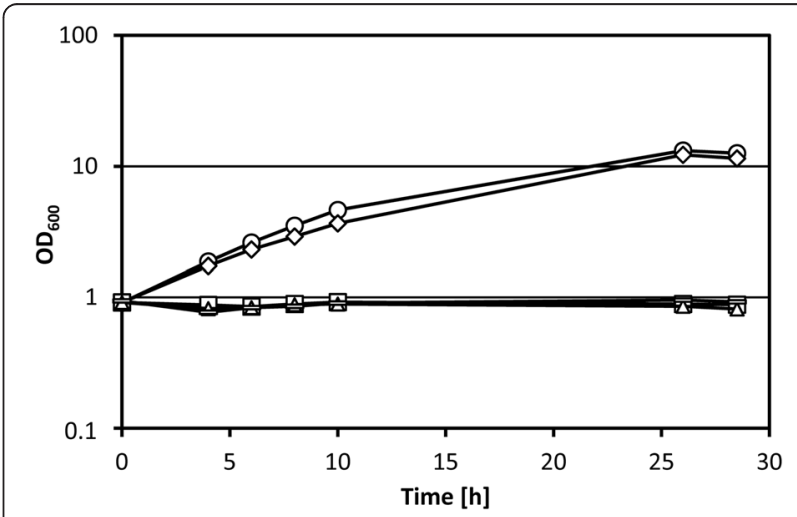

Fig. 4 Growth of C. glutamicum mutants with ethanol. Growth of C. glutamicum WT (diamonds), Tol1 (triangles), T0755 (circles), T2840 (bars) and T0755 + 2840 (squares) in minimal medium supplemented with $1.5 \%$ ethanol as sole source of carbon and energy. Means and standard deviations of two independent cultures are shown 
of the methylotroph bacterium Bacillus methanolicus is dependent on the activities of the methanol dehydrogenase Mdh and the enzymes 3-hexulose 6-phosphate synthase (Hps) and 6-phospho-3-hexuloisomerase (Phi), responsible for utilization of formaldehyde [43]. However, in C. glutamicum deletion of adhA did not affect methanol tolerance although the encoded alcohol dehydrogenase oxidizes methanol to formaldehyde. Moreover, the evolved strain Tol1 did not show increased tolerance to the methanol metabolites formaldehyde and formate. Notably, growth of Tol1 was not impaired as drastic as C. glutamicum WT at methanol concentrations up to $120 \mathrm{mM}$, while the maximal methanol concentration tolerated was similar for WT and Toll. This argued that the sharp decline of the growth rate observed for C. glutamicum WT may be caused by methanol itself or compounds derived from methanol in reactions other than those of the linear methanol oxidation pathway. Direct toxic effects of methanol are known to be based on its hydrophobic character, which is affecting the stability of the cellular membrane [5]. It has been shown that the tolerance to other membrane affecting alcohols like butanol could be increased by mutations in genes corresponding to membrane stability [44]. However, the identified mutations increasing methanol tolerance in C. glutamicum were not found in genes affecting membrane stability. The second component of the response of C. glutamicum to methanol evident at higher methanol concentrations may be due to membrane damages by methanol or toxicity of formate and formaldehyde.

The finding that mutations affecting $\mathrm{O}$-acetylhomoserine sulfhydrolase MetY and CoA transferase Cat were sufficient to explain increased methanol tolerance of C. glutamicum Tol1 indicated that their enzymatic (side) reactions contribute to methanol toxicity and are prominent in particular at concentrations up to about $250 \mathrm{mM}$. In C. glutamicum, methionine functions as methyl donor and is synthesized either by transsulfuration or by direct sulfhydrylation catalyzed by MetY [45]. In the reaction of MetY, O-acetylhomoserine is directly converted to homocysteine using sulfide [46]. In addition, MetY is also able to convert $\mathrm{O}$-acetylhomoserine to methionine using methanethiol (Reaction: O-acetyl-homoserine + methanethiol $<=>$ methionine + acetate) [47]. Methanethiol is the thiol equivalent of methanol and it has been shown that MetY from Corynebacterium acetophilum and Saccharomyces cerevisiae accepts methanol and other short-chain alcohols as substrates in addition to sulfide and methanethiol [48]. The MetY-catalyzed alkylation of $\mathrm{O}$-acetylhomoserine with methanol yields $\mathrm{O}$-methylhomoserine and acetate (Reaction: O-acetylhomoserine + methanol $<=>$ O-methyl-homoserine + acetate). Since $\mathrm{O}$-methylhomoserine is known to inhibit growth of $E$. coli and other microorganisms [49], this MetY-catalyzed reaction may contribute to the methanol toxicity of C. glutamicum. Strain Tol1 synthesized a variant of MetY (A165T) and showed higher RNA levels of met $Y$ in complex medium with added methanol than C. glutamicum WT (Additional file 1: Table S1). Albeit strain Tol1 was not further investigated since O-acetylhomoserine could not be obtained, the location of A165 close to a substrate-cofactor binding motif predicted by CDD-alignment [40] may indicate that binding of the cofactor pyridoxal-5-phosphate, which is essential for the function of MetY [46], and MetY activity are affected. While MetY clearly contributed to methanol toxicity in C. glutamicum, the biochemical mechanism remains to be explored by combining in vitro and in vivo approaches such as metabolomics and structure-function analyses of MetY and Met $Y^{\mathrm{A} 165 \mathrm{~T}}$.

The second mutation contributing to increased methanol tolerance of strain Tol1 led to truncation of CoA transferase Cat. Cat transfers CoA between acetyl-, propionyl- and succinyl-CoA thioesters and the respective free acids [41]. Whereas it is known that cat is highly and constitutively expressed [41], its function remains elusive since only a role in acetate and propionate catabolism in the absence of acetate kinase Ack and phosphotransacetylase Pta during co-consumption with glucose was found $[41,50]$. Both the lack of Cat activity due to deletion of cat as well as its truncation due to the SNP present in strain Tol1 increased the methanol tolerance. Thus, either the activity of CoA transfer between the acids acetate, propionate or succinate and the respective thioesters [41], or enzymatic side activity of Cat result in reduced growth in the presence of methanol. Several side reactions appear possible and although not all of them have been documented, in other organisms CoA transferases may have activity as acetyl-CoA hydrolases, as alcohol acetyltransferases or may form methyl-CoA. Alcohol acetyltransferases e.g. from Saccharomyces uvarum catalyze the transfer of the acetyl moiety from acetyl-CoA to methanol resulting in methyl acetate ester [51]. Methyl-Coenzyme $M$ is an intermediate in methanogenic archaea like Methanosarcina barkeri and is formed by methanol:coenzyme $M$ methyltransferase [52]. Due to structural similarities of the coenzymes A and $\mathrm{M}$, an analogous reaction of methanol with coenzyme A might be possible. Future metabolomics and structure-function analyses are necessary to determine if Cat from C. glutamicum WT, but not from Tol1, also possesses activity as acetyl-CoA hydrolase, alcohol acetyltransferase or for generation of methyl-CoA and if the resulting intermediates are growth inhibitory. This also pertains to the finding that truncation of Cat as well as the absence of Cat precluded use of ethanol as sole source of carbon and energy. It is not clear whether 
increased methanol tolerance and the inability to use ethanol are interdependent or arose by chance as consequence of Cat truncation.

Growth of C. glutamicum with ethanol involves oxidation of ethanol to acetate and requires activation by the Pta/Ack-system and operation of the glyoxylate cycle [31]. Thus, acetyl-CoA is an essential intermediate of ethanol metabolism and its concentration would be reduced if truncated Cat were active as acetyl-CoA hydrolase. Alternatively, induction of genes $a c k, p t a, a c e A$ and $a c e B$ by ethanol [31], which is as high as induction by acetate [50,53] or methanol [20] may be impaired by truncated Cat. Indeed, induction of these genes was not observed in Tol1 (Additional file 1: Table S2). The genes ack, pta, ace $A$ are directly repressed by $\operatorname{RamB}[54,55]$ and activated by RamA [56, 57]. AceA and $a c e B$ are directly repressed by GlxR, but regulation of the pta-ack operon by GlxR has not yet been demonstrated in vivo. GlxR and SugR indirectly control these genes by regulation of ramA expression [56]. C. glutamicum mutants lacking RamA cannot grow with acetate or ethanol as sole carbon sources [55]. The physiological trigger for the regulation of the ethanol and acetate metabolism is still unknown, but has been inferred as acetyl-CoA or a derivative thereof based on missing induction of genes from the acetate metabolism as result of interrupted ack and pta genes [53]. Thus, the inducer may not be synthesized or may be degraded by truncated Cat.

The identified mutations increasing methanol tolerance of C. glutamicum are relevant to strain development for biotechnological applications either using methanol as (co-)substrate or using growth substrates containing methanol as impurity. The latter was already shown to be of biotechnological relevance since C. glutamicum engineered to utilize glycerol for growth and amino acid production readily used pure glycerol, but not all technical qualities of glycerol $[29,30]$. Crude glycerol is a by-product of biodiesel production by transesterification of plant fats with methanol and often contains residual methanol [58]. Producer strains carrying the mutations of met $Y$ and cat may show improved performance in processes based on crude glycerol.

\section{Conclusions}

A C. glutamicum strain with increased methanol tolerance was selected by adaptive laboratory evolution. Genome sequencing of this strain identified two SNPs leading to amino acid substitution A165T of the Oacetylhomoserine sulfhydrolase MetY and truncation (Q342*) of CoA transferase by 161 amino acids. Introduction of these mutations into the wild type improved tolerance to methanol to the same level as observed with the selected mutant Tol1. Thus, these two mutations were sufficient to explain increased methanol tolerance of the selected strain. Introduction of these mutations into producer strains may facilitate production processes when using methanol as (co-)substrate or using growth substrates containing methanol as impurity.

\section{Methods}

\section{Microorganisms and cultivation conditions}

The strains and plasmids used in this study are listed in Table 1 . The E. coli strain DH5 $\alpha$ was used as a standard cloning host [59].

Cultivation of E. coli strains was carried out in LuriaBertani broth complex medium (LB) aerobically on a rotary shaker $(120 \mathrm{rpm})$ at $37^{\circ} \mathrm{C}$. C. glutamicum was cultivated aerobically on a rotary shaker $(120 \mathrm{rpm})$ at $30{ }^{\circ} \mathrm{C}$. Growth experiments with C. glutamicum were also performed in the microbioreactor system BioLector $(\mathrm{m} 2 \mathrm{p}$ labs; Baesweiler, Germany) using FlowerPlate microtiter plates (m2p labs; Baesweiler, Germany). The growth conditions were set to $1100 \mathrm{rpm}, 30{ }^{\circ} \mathrm{C}, 85 \%$ humidity and backscatter gain 20.. LB medium supplemented with $50 \mathrm{mM}$ glucose or bovine heart infusion (BHI) medium were used for precultures. Growth experiments with $C$. glutamicum were performed in the minimal medium mCGXII [21], a modified CGXII medium [60]. The medium was supplemented with $100 \mathrm{mM}$ glucose as carbon and energy source. For selection of clones carrying the plasmids pVWEx1 or pK19mobsacB and their derivatives, kanamycin was added to the medium in a concentration of $25 \mu \mathrm{g} \mathrm{ml}^{-1}$.

\section{Adaptive laboratory evolution}

In order to obtain methanol tolerant mutants, C. glutamicum (pVWEx1) was repeatedly cultivated in selective mCGXII medium containing $100 \mathrm{mM}$ glucose, kanamycin and $120 \mathrm{mM}$ methanol using two independent cultures. This is the highest concentration in the first concentration range, in which a sharp decrease of the growth rate was observed. The cultures were serially passed to fresh medium in regular intervals for about 50 generations.

The cultures grown in selective medium were subsequently cultivated on BHI plates with kanamycin. Single colonies from these plates were again cultivated in BHI $\mathrm{Kan}_{25}$ liquid medium followed by analysis of the methanol tolerance to exclude non-genetic adaption to methanol.

\section{DNA preparation, manipulation and transformation}

Plasmid isolation, molecular cloning and transformation of $E$. coli as well as electrophoresis were performed using standard procedures [61]. Transformation of C. glutamicum was performed by electroporation as described previously [62]. Chromosomal DNA of C. glutamicum was prepared as formerly described [63]. PCR experiments were performed using GoTaq DNA polymerase (Promega) 
Table 1 Strains and plasmids used in this study

\begin{tabular}{|c|c|c|}
\hline Strain or plasmid & Relevant characteristics & Reference or source \\
\hline E. coli DH5a & 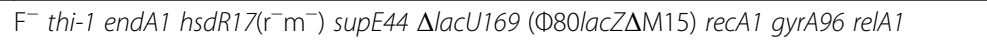 & [59] \\
\hline \multicolumn{3}{|l|}{ C. glutamicum strains } \\
\hline WT & Wild-type strain ATCC 13032 & American Type Culture Collection \\
\hline Tol1 & Methanol tolerant strain derived from C. glutamicum WT carrying the vector pWWEx1 & This study \\
\hline Tol2 & Methanol tolerant strain derived from C. glutamicum WT carrying the vector pWWEx1 & This study \\
\hline$\triangle a d h A$ & in-frame deletion of the adhA gene (cg3107) of C. glutamicum WT & [21] \\
\hline$\Delta r a m A$ & in-frame disruption of the ramA gene (cg2831) of C. glutamicum WT & [55] \\
\hline T0198 & 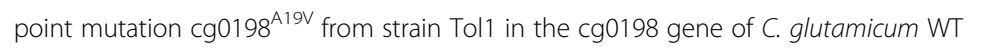 & This study \\
\hline T0755 & point mutation $\mathrm{cg}^{0755^{\mathrm{A} 165 \mathrm{~T}}}$ from strain Tol1 in the $\mathrm{cg} 0755$ gene of $\mathrm{C}$. glutamicum WT & This study \\
\hline $\mathrm{T} 1245$ & point mutation $\mathrm{cg} 1245^{\mathrm{L} 238 \mathrm{~S}}$ from strain Tol1 in the $\mathrm{cg} 1245$ gene of C. glutamicum WT & This study \\
\hline T2204 & point mutation $\mathrm{cg} 2204^{\mathrm{D} 67 \mathrm{H}}$ from strain Tol1 in the $\mathrm{cg} 2204$ gene of C. glutamicum WT & This study \\
\hline T2840 & point mutation $\mathrm{cg} 2840^{\mathrm{Q} 342^{*}}$ from strain Tol1 in the $\mathrm{cg} 2840$ gene of C. glutamicum WT & This study \\
\hline T0755 + 2840 & $\begin{array}{l}\text { Combination of the point mutations from strain Tol1 in the cg0755 and cg2840 genes } \\
\text { of C. glutamicum WT }\end{array}$ & This study \\
\hline$\Delta c a t$ & $\begin{array}{l}\text { in-frame deletion of the cat gene (cg2840) of C. glutamicum WT lacking both the } \\
\mathrm{N} \text { - and C-terminal regions of Cat }\end{array}$ & [41] \\
\hline \multicolumn{3}{|l|}{ Plasmids } \\
\hline pK19mobsacB & $\begin{array}{l}\mathrm{Km}^{\mathrm{r}} \text {, mobilizable E. coli vector for the construction of insertion and deletion mutants } \\
\text { of } C \text {. glutamicum (oriV, sacB, lacZa) }\end{array}$ & [65] \\
\hline pK19mobsacB-T0198 & $\begin{array}{l}\mathrm{Km}^{r} \text {, pK19mobsacB with the construct for a base exchange from } C \text { to } T \text { at position } \\
56 \text { in cg0198 }\end{array}$ & This study \\
\hline pK19mobsacB-T0755 & $\begin{array}{l}\mathrm{Km}^{\mathrm{r}} \text {, pK19mobsacB with the construct for a base exchange from } \mathrm{G} \text { to } \mathrm{A} \text { at position } \\
493 \text { in cg0755 }\end{array}$ & This study \\
\hline pK19mobsacB-T1245 & $\begin{array}{l}\mathrm{Km}^{r} \text {, pK19mobsacB with the construct for a base exchange from } T \text { to } C \text { at position } \\
983 \text { in cg1245 }\end{array}$ & This study \\
\hline pK19mobsacB-T2204 & $\begin{array}{l}\text { Kmr', pK19mobsacB with the construct for a base exchange from } G \text { to } C \text { at position } \\
199 \text { in cg2204 }\end{array}$ & This study \\
\hline pK19mobsacB-T2840 & $\begin{array}{l}\mathrm{Km}^{\mathrm{r}} \text {, pK19mobsacB with the construct for a base exchange from } \mathrm{C} \text { to } \mathrm{T} \text { at position } \\
1024 \text { in cg2840 }\end{array}$ & This study \\
\hline pWWEx1 & $\mathrm{Km}^{r} ;$ C. glutamicum/E. coli shuttle vector ( $P_{\text {tac }}$ lac $\left.\right|^{q}$ oriV C.g. ori $\left._{E . c .}\right)$ & [71] \\
\hline
\end{tabular}

or KOD Hot Start polymerase (Novagen) with oligonucleotides obtained from Metabion (listed in Additional file 1: Table S4). All restriction enzymes and polynucleotide kinase were obtained from Fermentas and used according to the manufacturer's instructions. Dephosphorylation and ligation were performed using the Rapid DNA Dephos \& Ligation Kit from Roche. Plasmids were isolated from $E$. coli using the QIAprep miniprep kit (Qiagen, Hilden, Germany). DNA sequencing was used to control all inserts of the plasmid constructs listed in Table 1.

\section{Construction of $C$. glutamicum mutant strains}

The single base substitutions in cg0198, cg0755, cg1245, cg2204 and cg2840 were constructed in C. glutamicum WT using the corresponding derivatives of plasmid pK19mobsacB as described previously [64]. These vectors carry the mutated basepairs flanked by native sequences upstream and downstream. The flanking regions were amplified from genomic DNA of C. glutamicum via PCR using corresponding primer pairs $\mathrm{A}$ and $\mathrm{B}$ as well as $\mathrm{C}$ and D (Additional file 1: Table S4). The resulting PCR products as well as primers $A$ and $D$ were used in the subsequent crossover PCR reaction. The resulting fusion product was phosphorylated with polynucleotide kinase and ligated into SmaI digested vector pK19mobsacB [65]. Point mutations were verified by amplification using the primers $\mathrm{A}$ and $\mathrm{D}$ and subsequent sequence analysis.

\section{Alcohol dehydrogenase enzyme assays}

Enzyme activity of AdhA was measured in crude cell extracts at $30{ }^{\circ} \mathrm{C}$ using a Shimadzu UV-1800 spectrophotometer by following the formation of NADH at $340 \mathrm{~nm}$ $\left(\Delta \varepsilon=6230 \mathrm{M}^{-1} \mathrm{~cm}^{-1}\right)$. Crude cell extracts were prepared using sonication treatment [21]. Buffers and assay conditions have been described previously [31] and the reaction was started by addition of $1 \mathrm{M}$ ethanol. 


\section{Analysis of formaldehyde degradation}

In vivo formaldehyde degradation assays were performed using resting cells. For this purpose, $50 \mathrm{ml}$ mCGXII medium without carbon source was inoculated from a $\mathrm{LB}$ culture to an $\mathrm{OD}_{600}$ of 1 and incubated in a $500 \mathrm{ml}$ baffled Erlenmeyer flask at $30{ }^{\circ} \mathrm{C}$ and $120 \mathrm{rpm}$. The assays were started by addition of $0.5 \mathrm{mM}$ formaldehyde. Measurement of formaldehyde concentrations was performed using a colorimetric method as described previously [21].

\section{Transcriptome analysis using DNA microarrays}

Gene expression analysis in C. glutamicum WT and strain Tol1 was performed after cultivation in LB or mCGXII medium. RNA was isolated during the mid log growth phase followed by synthesis of fluorescently labeled cDNA from RNA, DNA microarray hybridization and gene expression analysis $[66,67]$. The data were normalized using the LOWESS approach. The significance of gene expression rates was determined using a $t$-test adjusted with the False Discovery Rate approach. Individual data points were not considered as significant if the signal to noise ratio of both channels was below 3, less than two third of the replicates showed regulation or the A-value was below 8. Furthermore, the adjusted p-value had to be higher than 0.05 and the genes needed to be regulated more than two-fold.

\section{Genome sequence analysis}

Libraries were prepared from isolated genomic DNA as described previously [68]. Sequencing of the libraries was performed on the Genome Analyze IIx platform (Illumina, San Diego, CA, USA) using a single read cluster generation kit $\mathrm{v} 4$ according to the manufacturer's instructions. 32 bp sequence reads were mapped to the genome sequence of C. glutamicum ATCC13032 [37] using the program SARUMAN [69]. The coverage was obtained by multiplying the read length by the respective read start. Perl programming language script implemented for Parsing of the read start information and calculation of coverage and read start numbers. Variants were considered to be significant if they possessed a frequency higher than $90 \%$ and coverage of at least 70 .

\section{Availability of supporting data}

All supporting data are included as additional files. Microarray data have also been deposited in NCBI's Gene Expression Omnibus [70] and are accessible through GEO Series accession number GSE71590 (http:// www.ncbi.nlm.nih.gov/geo/query/acc.cgi?acc=GSE71590).

\section{Additional file}

Additional file 1: Table S1. Genome-wide comparison of mRNA levels between the strains Tol1 and C. glutamicum WT(pWWEx1) during growth in LB complex medium. Table S2. Genome-wide comparison of mRNA levels in Tol1 and C. glutamicum wild type cultivated in minimal medium with $100 \mathrm{mM}$ glucose in presence or absence of methanol. Table S3. Mutations detected in strain Tol1 and a control strain in comparison to the C. glutamicum wild type genome sequence NC_006958.1. Table S4. Oligonucleotides used in this study. Figure S1. Growth in the presence of formaldehyde (A) and formaldehyde degradation (B). Figure S2. Growth of C. glutamicum wild type with propionate and methanol. Figure S3. Growth of C. glutamicum $\Delta$ ramA with methanol. Figure S4. Growth of C. glutamicum $\Delta$ cat with methanol. Figure S5. Biomass formation of C. glutamicum wild type, Tol1, T2840 and $\Delta$ cat in minimal medium with ethanol as sole carbon source. (PDF $919 \mathrm{~kb}$ )

\section{Abbreviations}

PQQ: Pyrroloquinoline quinone; AdhA: Alcohol dehydrogenase; Ald: Acetaldehyde dehydrogenase; FadH: Mycothiol-dependent formaldehyde dehydrogenase; SNP: Single nucleotide polymorphism; CoA: Coenzyme A; CDD: Conserved domain database.

\section{Competing interests}

The authors declare that they have no competing interests.

\section{Authors' contributions}

VFW and LL designed the experiments. $L L$ conducted the experiments, analyzed the results and wrote the manuscript. VFW reviewed and revised the manuscript. Both authors read and approved the final manuscript.

\section{Acknowledgements}

We would like to thank Jörn Kalinowski, Christian Rückert and Andreas Albersmeier for genome sequence analysis. We acknowledge support of the publication fee by Deutsche Forschungsgemeinschaft and the Open Access Publication Funds of Bielefeld University. This work was funded by SynMet, a 09-EuroSYNBIO-FP-023 project funded in part by DFG through grant WE 2320/2-1.

Received: 15 April 2015 Accepted: 8 October 2015

Published online: 16 October 2015

\section{References}

1. Yang M, Nightingale PD, Beale R, Liss PS, Blomquist B, Fairall C. Atmospheric deposition of methanol over the Atlantic Ocean. Proc Natl Acad Sci U S A. 2013;110(50):20034-9.

2. von Dahl CC, Havecker M, Schlogl R, Baldwin IT. Caterpillar-elicited methanol emission: a new signal in plant-herbivore interactions? Plant J. 2006;46(6):948-60

3. Jacob DJ, Field BD, Li QB, Blake DR, de Gouw J, Warneke C, et al. Global budget of methanol: Constraints from atmospheric observations. J Geophys Res-Atmos. 2005;110:D08303.

4. Schink B, Zeikus JG. Microbial Methanol Formation - a Major End Product of Pectin Metabolism. Curr Microbiol. 1980:4(6):387-9.

5. Sonmez M, Ince HY, Yalcin O, Ajdzanovic V, Spasojevic I, Meiselman HJ, et al. The effect of alcohols on red blood cell mechanical properties and membrane fluidity depends on their molecular size. PLoS One. 2013;8(9):e76579

6. Gustafson C, Tagesson C. Influence of organic solvent mixtures on biological membranes. Br J Ind Med. 1985;42(9):591-5.

7. Teng S, Beard K, Pourahmad J, Moridani M, Easson E, Poon R, et al. The formaldehyde metabolic detoxification enzyme systems and molecular cytotoxic mechanism in isolated rat hepatocytes. Chem Biol Interact. 2001;130-132(1-3):285-96.

8. Chang CC, Gershwin ME. Perspectives on formaldehyde toxicity: separating fact from fantasy. Regul Toxicol Pharmacol. 1992;16(2):150-60.

9. Tephly TR. The toxicity of methanol. Life sciences. 1991:48(11):1031-41.

10. Nicholls $P$. The effect of formate on cytochrome aa3 and on electron transport in the intact respiratory chain. Biochim Biophys Acta. 1976;430(1):13-29. 
11. Liesivuori J, Savolainen H. Methanol and formic acid toxicity: biochemical mechanisms. Pharmacol Toxicol. 1991;69(3):157-63.

12. Dobrzynska I, Skrzydlewska E, Kasacka I, Figaszewski Z. Protective effect of $\mathrm{N}$-acetylcysteine on rat liver cell membrane during methanol intoxication. J Pharm Pharmacol. 2000;52(5):547-52.

13. Parthasarathy NJ, Kumar RS, Manikandan S, Devi RS. Methanol-induced oxidative stress in rat lymphoid organs. J Occup Health. 2006;48(1):20-7.

14. Vorholt JA. Cofactor-dependent pathways of formaldehyde oxidation in methylotrophic bacteria. Arch Microbiol. 2002;178(4):239-49.

15. Afolabi PR, Mohammed F, Amaratunga K, Majekodunmi O, Dales SL, Gill R, et al. Site-directed mutagenesis and X-ray crystallography of the PQQcontaining quinoprotein methanol dehydrogenase and its electron acceptor, cytochrome $c(L)$. Biochemistry. 2001;40(33):9799-809.

16. Wagner FW, Burger AR, Vallee BL. Kinetic properties of human liver alcohol dehydrogenase: oxidation of alcohols by class I isoenzymes. Biochemistry. 1983;22(8):1857-63.

17. Aggelis G, Fakas S, Melissis S, Clonis YD. Growth of Candida boidinii in a methanol-limited continuous culture and the formation of methanoldegrading enzymes. J Biotechnol. 1999;72(1-2):127-39.

18. Wendisch VF. Microbial production of amino acids and derived chemicals: synthetic biology approaches to strain development. Curr Opin Biotechnol. 2014;30:51-8.

19. Mitsuhashi S. Current topics in the biotechnological production of essential amino acids, functional amino acids, and dipeptides. Curr Opin Biotechnol. 2014:26:38-44

20. Witthoff S, Muhlroth A, Marienhagen J, Bott M. C1 metabolism in Corynebacterium glutamicum: an endogenous pathway for oxidation of methanol to carbon dioxide. Appl Environ Microbiol. 2013;79(22):6974-83.

21. Lessmeier $L$, Hoefener M, Wendisch VF. Formaldehyde degradation in Corynebacterium glutamicum involves acetaldehyde dehydrogenase and mycothiol-dependent formaldehyde dehydrogenase. Microbiology. 2013:159(Pt 12):2651-62.

22. Witthoff $\mathrm{S}$, Eggeling $\mathrm{L}$, Bott M, Polen T. Corynebacterium glutamicum harbours a molybdenum cofactor-dependent formate dehydrogenase which alleviates growth inhibition in the presence of formate. Microbiology. 2012;158(Pt 9):2428-39.

23. Sato S, Kitamoto D, Habe H. Chemical mutagenesis of Gluconobacter frateurii to construct methanol-resistant mutants showing glyceric acid production from methanol-containing glycerol. J Biosci Bioeng. 2014;117(2):197-9.

24. Brautaset $T$, Jakobsen $O M$, Josefsen KD, Flickinger MC, Ellingsen TE. Bacillus methanolicus: a candidate for industrial production of amino acids from methanol at 50 degrees C. Appl Microbiol Biotechnol. 2007;74(1):22-34

25. Schrader J, Schilling M, Holtmann D, Sell D, Filho MV, Marx A, et al. Methanol-based industrial biotechnology: current status and future perspectives of methylotrophic bacteria. Trends Biotechnol. 2009;27(2):107-15

26. Naerdal I, Pfeifenschneider J, Brautaset T, Wendisch VF. Methanol-based cadaverine production by genetically engineered Bacillus methanolicus strains. Microb Biotechnol. 2015;8(2):342-50.

27. Witthoff S, Schmitz K, Niedenfuhr S, Noh K, Noack S, Bott M, et al. Metabolic engineering of Corynebacterium glutamicum for methanol metabolism. Appl Environ Microbiol. 2015;81(6):2215-25.

28. Leßmeier L, Pfeifenschneider J, Carnicer M, Heux S, Portais JC, Wendisch VF. Production of carbon-13-labeled cadaverine by engineered Corynebacterium glutamicum using carbon-13-labeled methanol as co-substrate Applied Microbiology and Biotechnology. 2015; in press.

29. Rittmann D, Lindner SN, Wendisch VF. Engineering of a glycerol utilization pathway for amino acid production by Corynebacterium glutamicum. Appl Environ Microbiol. 2008;74(20):6216-22.

30. Meiswinkel TM, Rittmann D, Lindner SN, Wendisch VF. Crude glycerol-based production of amino acids and putrescine by Corynebacterium glutamicum. Bioresour Technol. 2013;145:254-8.

31. Arndt A, Auchter M, Ishige T, Wendisch VF, Eikmanns BJ. Ethanol catabolism in Corynebacterium glutamicum. J Mol Microbiol Biotechnol. 2008;15(4):222-33.

32. Kotrbova-Kozak A, Kotrba P, Inui M, Sajdok J, Yukawa H. Transcriptionally regulated $a d h A$ gene encodes alcohol dehydrogenase required for ethanol and n-propanol utilization in Corynebacterium glutamicum R. Appl Microbiol Biotechnol. 2007;76(6):1347-56.
33. Claes WA, Puhler A, Kalinowski J. Identification of two prpDBC gene clusters in Corynebacterium glutamicum and their involvement in propionate degradation via the 2-methylcitrate cycle. J Bacteriol. 2002;184(10):2728-39.

34. Cramer A, Eikmanns BJ. RamA, the transcriptional regulator of acetate metabolism in Corynebacterium glutamicum, is subject to negative autoregulation. J Mol Microbiol Biotechnol. 2007;12(1-2):51-9.

35. Auchter M, Arndt A, Eikmanns BJ. Dual transcriptional control of the acetaldehyde dehydrogenase gene ald of Corynebacterium glutamicum by RamA and RamB. J Biotechnol. 2009;140(1-2):84-91.

36. Arndt A, Eikmanns BJ. The alcohol dehydrogenase gene adhA in Corynebacterium glutamicum is subject to carbon catabolite repression. J Bacteriol. 2007;189(20):7408-16.

37. Kalinowski J, Bathe B, Bartels D, Bischoff N, Bott M, Burkovski A, et al. The complete Corynebacterium glutamicum ATCC 13032 genome sequence and its impact on the production of L-aspartate-derived amino acids and vitamins. J Biotechnol. 2003;104(1-3):5-25.

38. Baumgart M, Unthan S, Ruckert C, Sivalingam J, Grunberger A, Kalinowski J, et al. Construction of a prophage-free variant of Corynebacterium glutamicum ATCC 13032 for use as a platform strain for basic research and industrial biotechnology. Appl Environ Microbiol. 2013;79(19):6006-15.

39. Komati Reddy G, Lindner SN, Wendisch VF. Metabolic Engineering of an ATP-Neutral Embden-Meyerhof-Parnas Pathway in Corynebacterium glutamicum: Growth Restoration by an Adaptive Point Mutation in NADH Dehydrogenase. Appl Environ Microbiol. 2015;81(6):1996-2005.

40. Marchler-Bauer A, Lu S, Anderson JB, Chitsaz F, Derbyshire MK, DeWeese-Scott C, et al. CDD: a Conserved Domain Database for the functional annotation of proteins. Nucleic Acids Res. 2011;39(Database issue):D225-9.

41. Veit A, Rittmann D, Georgi T, Youn JW, Eikmanns BJ, Wendisch VF. Pathway identification combining metabolic flux and functional genomics analyses: acetate and propionate activation by Corynebacterium glutamicum. J Biotechnol. 2009;140(1-2):75-83.

42. Garcia MX, Roberts C, Alexander H, Stewart AM, Harwood A, Alexander S, et al. Methanol and acriflavine resistance in Dictyostelium are caused by loss of catalase. Microbiology. 2002;148(Pt 1):333-40.

43. Jakobsen $\mathrm{OM}$, Benichou A, Flickinger MC, Valla S, Ellingsen TE, Brautaset $T$. Upregulated transcription of plasmid and chromosomal ribulose monophosphate pathway genes is critical for methanol assimilation rate and methanol tolerance in the methylotrophic bacterium Bacillus methanolicus. J Bacteriol. 2006;188(8):3063-72.

44. Reyes LH, Abdelaal AS, Kao KC. Genetic determinants for n-butanol tolerance in evolved Escherichia coli mutants: cross adaptation and antagonistic pleiotropy between n-butanol and other stressors. Appl Environ Microbiol. 2013;79(17):5313-20.

45. Hwang BJ, Yeom HJ, Kim Y, Lee HS. Corynebacterium glutamicum utilizes both transsulfuration and direct sulfhydrylation pathways for methionine biosynthesis. J Bacteriol. 2002;184(5):1277-86.

46. Hwang BJ, Park SD, Kim Y, Kim P, Lee HS. Biochemical analysis on the parallel pathways of methionine biosynthesis in Corynebacterium glutamicum. J Microbiol Biotechnol. 2007;17(6):1010-7.

47. Bolten CJ, Schroder H, Dickschat J, Wittmann C. Towards methionine overproduction in Corynebacterium glutamicum-methanethiol and dimethyldisulfide as reduced sulfur sources. J Microbiol Biotechnol. 2010;20(8):1196-203.

48. Murooka Y, Kakihara K, Miwa T, Seto K, Harada T. O-alkylhomoserine synthesis catalyzed by O-acetylhomoserine sulfhydrylase in microorganisms. J Bacteriol. 1977:130(1):62-73.

49. Roblin RO, Lampen JO, English JP, Cole QP, Vaughan JR. Studies in Chemotherapy.8. Methionine and Purine Antagonists and Their Relation to the Sulfonamides. J Am Chem Soc. 1945;67(2):290-4.

50. Reinscheid DJ, Schnicke S, Rittmann D, Zahnow U, Sahm H, Eikmanns BJ. Cloning, sequence analysis, expression and inactivation of the Corynebacterium glutamicum pta-ack operon encoding phosphotransacetylase and acetate kinase. Microbiology. 1999;145(Pt 2):503-13.

51. Yoshioka K, Hashimoto N. Ester Formation by Alcohol Acetyltransferase from Brewers-Yeast. Agr Biol Chem Tokyo. 1981;45(10):2183-90

52. Kruer M, Haumann M, Meyer-Klaucke W, Thauer RK, Dau H. The role of zinc in the methylation of the coenzyme $M$ thiol group in methanol:coenzyme $\mathrm{M}$ methyltransferase from Methanosarcina barkeri. Eur J Biochem. 2002;269(8):2117-23

53. Wendisch VF, Spies M, Reinscheid DJ, Schnicke S, Sahm H, Eikmanns BJ. Regulation of acetate metabolism in Corynebacterium glutamicum: 
transcriptional control of the isocitrate lyase and malate synthase genes. Arch Microbiol. 1997;168(4):262-9.

54. Gerstmeir R, Cramer A, Dangel P, Schaffer S, Eikmanns BJ. RamB, a novel transcriptional regulator of genes involved in acetate metabolism of Corynebacterium glutamicum. J Bacteriol. 2004;186(9):2798-809.

55. Cramer A, Gerstmeir R, Schaffer S, Bott M, Eikmanns BJ. Identification of RamA, a novel LuxR-type transcriptional regulator of genes involved in acetate metabolism of Corynebacterium glutamicum. J Bacteriol. 2006;188(7):2554-67.

56. Toyoda K, Teramoto H, Gunji W, Inui M, Yukawa H. Involvement of regulatory interactions among global regulators $G \mid x R$, SugR, and RamA in expression of ramA in Conynebacterium glutamicum. J Bacteriol. 2013;195(8):1718-26.

57. Park SY, Moon MW, Subhadra B, Lee JK. Functional characterization of the glxR deletion mutant of Corynebacterium glutamicum ATCC 13032: involvement of GIxR in acetate metabolism and carbon catabolite repression. FEMS Microbiol Lett. 2010;304(2):107-15.

58. Chatzifragkou A, Papanikolaou S. Effect of impurities in biodiesel-derived waste glycerol on the performance and feasibility of biotechnological processes. Appl Microbiol Biotechnol. 2012;95(1):13-27.

59. Hanahan D. Studies on transformation of Escherichia coli with plasmids. J Mol Biol. 1983;166(4):557-80.

60. Keilhauer C, Eggeling L, Sahm H. Isoleucine synthesis in Corynebacterium glutamicum: molecular analysis of the ilvB-ilvN-ilvC operon. J Bacteriol. 1993;175(17):5595-603.

61. Sambrook J, Russell D. Molecular Cloning. A Laboratory Manual, 3rd Edition. Cold Spring Harbor, NY: Cold Spring Harbor Laboratoy Press; 2001.

62. Tauch A, Kirchner O, Loffler B, Gotker S, Puhler A, Kalinowski J. Efficient electrotransformation of Corynebacterium diphtheriae with a mini-replicon derived from the Corynebacterium glutamicum plasmid pGA1. Curr Microbiol. 2002;45(5):362-7.

63. Eikmanns BJ, Thum-Schmitz N, Eggeling L, Ludtke KU, Sahm H. Nucleotide sequence, expression and transcriptional analysis of the Corynebacterium glutamicum gltA gene encoding citrate synthase. Microbiology. 1994;140(Pt 8):1817-28.

64. Peters-Wendisch PG, Wendisch VF, de Graaf AA, Eikmanns BJ, Sahm H. C3-carboxylation as an anaplerotic reaction in phosphoenolpyruvate carboxylase-deficient Corynebacterium glutamicum. Arch Microbiol. 1996;165(6):387-96.

65. Schäfer A, Tauch A, Jäger W, Kalinowski J, Thierbach G, Puhler A. Small mobilizable multi-purpose cloning vectors derived from the Escherichia coli plasmids pK18 and pK19: selection of defined deletions in the chromosome of Corynebacterium glutamicum. Gene. 1994;145(1):69-73.

66. Polen T, Schluesener D, Poetsch A, Bott M, Wendisch VF. Characterization of citrate utilization in Corynebacterium glutamicum by transcriptome and proteome analysis. FEMS Microbiol Lett. 2007;273(1):109-19.

67. Wendisch VF. Genome-wide expression analysis in Conynebacterium glutamicum using DNA microarrays. J Biotechnol. 2003;104(1-3):273-85.

68. Milse J, Petri K, Ruckert C, Kalinowski J. Transcriptional response of Corynebacterium glutamicum ATCC 13032 to hydrogen peroxide stress and characterization of the OxyR regulon. J Biotechnol. 2014;190:40-54.

69. Blom J, Jakobi T, Doppmeier D, Jaenicke S, Kalinowski J, Stoye J, et al. Exact and complete short-read alignment to microbial genomes using Graphics Processing Unit programming. Bioinformatics. 2011;27(10):1351-8.

70. Edgar R, Domrachev M, Lash AE. Gene Expression Omnibus: NCBI gene expression and hybridization array data repository. Nucleic Acids Res. 2002:30(1):207-10.

71. Peters-Wendisch PG, Schiel B, Wendisch VF, Katsoulidis E, Mockel B, Sahm H, et al. Pyruvate carboxylase is a major bottleneck for glutamate and lysine production by Corynebacterium glutamicum. J Mol Microbiol Biotechnol. 2001;3(2):295-300.

\section{Submit your next manuscript to BioMed Central and take full advantage of:}

- Convenient online submission

- Thorough peer review

- No space constraints or color figure charges

- Immediate publication on acceptance

- Inclusion in PubMed, CAS, Scopus and Google Scholar

- Research which is freely available for redistribution

Submit your manuscript at www.biomedcentral.com/submit 